Sof t war e- Def i ned Vehi cul ar Net wor ks: Archi t ect ure, Al gor i thms, and Appl i cat i ons: Part 1

\begin{tabular}{|l|l|}
\hline 著者 & $\begin{array}{l}\text { HAN Guangj i e, GUl ZAN Nohsen, BI Yuanguo, LUAN } \\
\text { Tom H. , OTA Kaor u, ZHOU Hai bo, GUl BENE Whel, } \\
\text { RAYES Ammar }\end{array}$ \\
\hline $\begin{array}{l}\text { j our nal or } \\
\text { publ i cat i on t i t l e }\end{array}$ & I EEE Communi cat i ons Nagazi ne \\
\hline vol une & 55 \\
\hline number & 7 \\
\hline page r ange & $78-79$ \\
\hline year & $2017-07-14$ \\
\hline URL & ht t p: //hdl . handl e. net /10258/00009570 \\
\hline
\end{tabular}




\section{SOFTWARE-Defined VeHICUlar NetWORKS: ARCHITECTURE, AlGORITHMS, AND APPLICATIONS: PART 1}

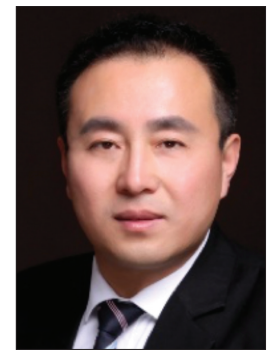

Guangjie Han

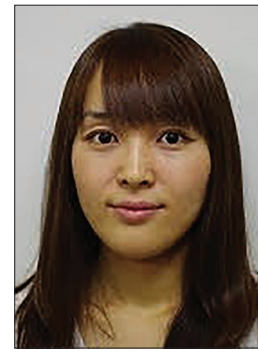

Kaoru Ota

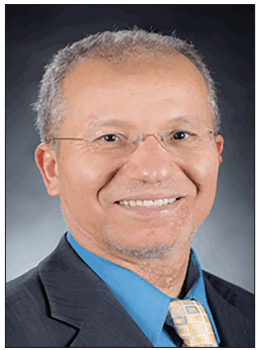

Mohsen Guizani

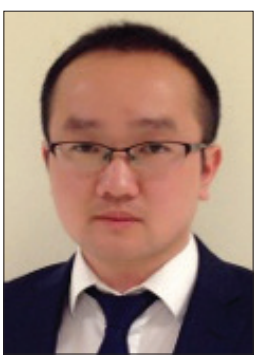

Haibo Zhou

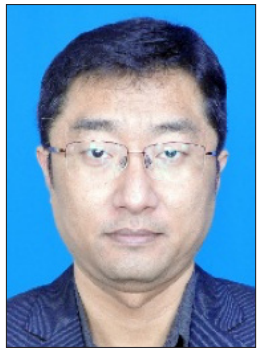

Yuanguo $\mathrm{Bi}$

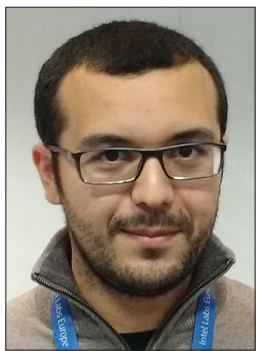

Wael Guibene

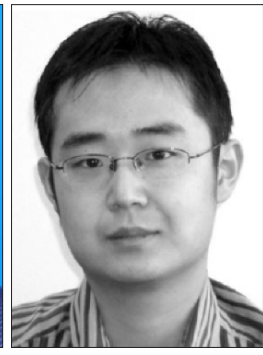

Tom H. Luan

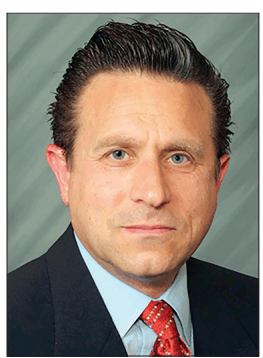

Ammar Rayes ith the ever more rapid development of wireless communications and the explosive usage of mobile electronics, vehicular networks have become an attainable technology to meet the imminent demands for improving traffic safety and efficiency. In addition, there is an increasing demand from traveling users to access the Internet through IP-enabled smart devices, which enables infotainment applications to have rapidly taken on an important role in the past few years. Even though several future architectures have been proposed and investigated, it has been very challenging to coordinate vehicular networks to efficiently facilitate applications with diverse quality of service (QoS) demands. Recently, software-defined networking (SDN) has been emerging as a promising paradigm to control the network in a systematic way. The flexibility and programmability of SDN, which are lacking in today's distributed wireless substrate, not only make it attractive to satisfy the QoS requirements of vehicular multimedia services, but also simplify the resource management in vehicular networks. Consequently, there is a need to conduct research to further investigate the standardization efforts and address challenging issues in the SDN enabled vehicular networks.

In this IEEE Communications Magazine Feature Topic (FT), the Guest Editors invited experts from research communities to discuss the architecture, applications, challenging ideas, and standardization efforts on enabling software-defined vehicular networks (SDVNs). After a rigorous review process, 15 papers have been selected to be published in this FT, eight of which are published in Part 1; the rest will be published later, in Part 2 of this FT.

In SDVNs, travelling vehicles may lose connectivity to the central SDN controller, which undermines the benefits provided by SDN. The first article, by S. Correia et al., "An Architecture for Hierarchical Software-Defined Vehicular Networks," describes the design and implementation of a vehicular-based hierarchical software-defined architecture that is dedicated to improving communication performance and efficiency in case of connectivity loss between moving vehicles and the controller. Simulation results demonstrate that the proposed approach performs better than simply falling back to traditional solutions.

To meet rigorous QoS requirements of multimedia services in vehicular networks, 5G mobile communication technologies are indispensable to future SDVN. X. Ge et al., in "5G Software Defined Vehicular Networks," propose a new vehicular network architecture that integrates 5G mobile communication technologies and SDN, in which fog cells are employed to flexibly cover vehicles and prevent frequent handover between vehicles and roadside units (RSUs). Simulation results reveal that there is a minimum transmission delay of 5G SDVNs under different vehicle densities, and the throughput of fog cells in 5G SDVNs can be improved compared to traditional proposals.

A heterogeneous vehicular network with the support of different access technologies is indispensable to provide reliable and ubiquitous mobile access. J. Wan et al., in "Scalable and Quick-Response Software Defined Vehicular Network Assisted by Mobile Edge Computing," propose an SDN-enabled network architecture to guarantee low-latency and high-reliability communications by integrating IEEE $802.11 \mathrm{p}$ and $5 \mathrm{G}$ radio access technologies in vehicular networks. The practical use case validates that the proposed architecture is 
able to meet application-specific requirements while maintaining network scalability.

To accommodate vehicular multimedia applications with diverse QoS requirements in various practical scenarios, it is imperative to exploit specific advantages of terrestrial networks, high-altitude communication platforms, and satellite communication systems. The next article, by N. Zhang et al., "Software Defined Space-Air-Ground Integrated Vehicular Networks: Challenges and Solutions," addresses these issues and proposes a software defined space-air-ground integrated network architecture to support various kinds of vehicular services in a seamless, efficient, and cost-effective manner, and the research directions in the proposed integrated vehicular network architecture are identified.

A layer-based top-down approach to systematically cater for security implications is essential to SDVN. A. Akhunzada et al., in "Toward Secure Software Defined Vehicular Networks: Taxonomy, Requirements, and Open Issues," present a top-down approach to address the security vulnerabilities, attacks, and challenges of each layer that is tightly dependent to anticipate secure emerging SDVNs. In addition, the requirements for securing SDVNs are also presented, and open research issues are discussed.

The fast varying network topology and high complexity of network infrastructure impose great challenges on supporting dynamic vehicular communications in 5G heterogeneous networks. X. Duan et al., in "SDN Enabled 5G-VANET: Adaptive Vehicle Clustering and Beamformed Transmission for Aggregated Traffic," present an SDN enabled 5G-VANET by adaptive vehicle clustering and beamformed transmission to accommodate dynamic aggregated traffic. Simulation results demonstrate that the SDN coordinated vehicle clustering and beamformed transmission can efficiently support fast varying traffic.

The distinctive characteristics of SDN are anticipated to facilitate vehicular communications. To explore the domain of SDVN, I. Yaqoob et al., in "Overcoming the Key Challenges of Establishing Vehicular Communication: Is SDN the Answer?," investigate, highlight, and report recent research advances in the SDVN paradigm. The key requirements that need to be met in SDVNs are outlined, and several research challenges are discussed as future research directions. In addition, they conclude that although SDN can improve management capabilities and address many challenges in the traditional VANET, integrating SDN and VANET will bring new challenges.

Integrating existing Wi-Fi networks into VANET is essential to the next generation vehicular networks. T. Q. Duong et al., in "Software Defined Architecture for VANET: A Testbed Implementation with Wireless Access Management," propose an SD-VANET testbed architecture that utilizes already deployed WiFi networks in the Istanbul Technical University campus. The proposed architecture is dedicated to minimize the necessity of changes in current network infrastructure, and avoids any change at the control-data plane interface.

In closing, we would like to thank all the people who have made significant contributions to this FT, including the contributing authors, the anonymous reviewers, and the IEEE Communications Magazine publications staff. We believe that the research results presented in this FT will stimulate further research and development ideas in vehicular networks.

\section{BIOGRAPHIES}

GUANGJIE HAN [S'01, M'05] is currently a professor with the Department of Information and Communication Systems, Hohai University, China. His current research interests include sensor networks, computer communications, mobile cloud computing, and multimedia communication and security. He has served on the Editorial Boards of 14 international journals, including IEEE Access and Telecommunication Systems. He has guest edited a number of Special Issues in IEEE journals and magazines. He is a member of ACM.

MOHSEN GuIZANI [S'85, M'89, SM'99, F'09] received his B.S, M.S., and Ph.D. from Syracuse University. He is currently a professor and the ECE Department Chair at the University of Idaho. His research interests include wireless communications/ mobile cloud computing, computer networks, security, and smart grid. He is the author of nine books and more 450 publications. He was the Chair of the IEEE Communications Society Wireless Technical Committee. He served as an IEEE Computer Society Distinguished Speaker.

YuANGUO Bı received his Ph.D. degree from Northeastern University, Shenyang, China, in 2010. He joined the School of Computer Science and Engineering, Northeastern University, China, as an associate professor in 2010. His current research interests focus on medium access control, QoS routing, multihop broadcast, mobility management in vehicular networks, as well as SDN enabled vehicular networks.

TOM H. LUAN received his Ph.D. degree from the University of Waterloo, Ontario, Canada, in 2012. Since December 2013, he has been a lecturer in mobile and apps with the School of Information Technology, Deakin University, Melbourne, Australia. His research mainly focuses on vehicular networking, wireless content distribution, peer-to peer networking, and mobile cloud computing.

KAORU OTA received Ph.D. degrees in computer science and engineering from the University of Aizu, Japan, in 2012. She is currently an assistant professor with the Department of Information and Electronic Engineering, Muroran Institute of Technology, Japan. She was also a research scientist with A3 Foresight Program (2011-2016) funded by the Japan Society for the Promotion of Sciences, NSFC of China, and NRF of Korea.

HAIBO ZHOU received his Ph.D. degree in information and communication engineering from Shanghai Jiao Tong University, China, in 2014. From 2014 to 2016 he was a postdoctoral research fellow with the Broadband Communications Research (BBCR) Group, University of Waterloo. He is currently a research associate in the BBCR Group. His current research interests include resource management and protocol design in cognitive radio networks and vehicular networks.

Ammar RaYes [S'85, M'91, SM'15] is a Distinguished Engineer focusing on the technology strategy for Cisco Services. His research interests include loT, network management NMS/OSS, machine learning, analytics, and security. He has authored three books, over 100 publications in refereed journals and conferences on advances in software and networking related technologies, and over 25 patents. He received B.S. and M.S. degrees from the University of Illinois at Urbana and his D.Sc. degree from Washington University, all in electrical engineering.

WAEL GUIBENE has been a research scientist at Intel Labs since June 2015. He was awarded his Ph.D. from Telecom ParisTech in July 2013. He also holds an M.Eng. and a Master's degree in telecommunications obtained in 2009 and 2010, respectively. He worked at Eurecom as a research engineer from 2010 to November 2013, and then joined Semtech to work on LoRa systems from 2013 to June 2015. His research activities include loT, 5G, and wireless communications. 